\title{
Clinical Characteristics of Patients with Intermittent Exotropia According to the Response to Short-term Prism Adaptation Test
}

\author{
Young In Yun ${ }^{1}$, Seong-Joon Kim, ${ }^{1,2}$ Jae Ho Jung, ${ }^{1,2}$ \\ ${ }^{I}$ Department of Ophthalmology, Seoul National University Hospital, Seoul, Korea \\ ${ }^{2}$ Department of Ophthalmology, Seoul National University College of Medicine, Seoul, Korea
}

Purpose: To evaluate the prevalence of the prism adaptation response in patients with intermittent exotropia (IXT) using the short-term prism adaptation test (PAT) and to assess factors associated with prism adaptation response in IXT patients.

Methods: A case-controlled retrospective analysis was performed on 113 patients with IXT without prior surgical treatment. Age, sex, visual acuity, refraction, stereoacuity, control scale, type of exotropia, history of occlusion, and presence of accompanying visual symptoms were recorded. Prism alternate cover test (PACT) was performed with fixation targets at $6 \mathrm{~m}$ and $1 / 3 \mathrm{~m}$. All patients underwent short-term PAT wearing prism glasses that offset the exodeviation previously measured by PACT. After 30 minutes, angle deviation was measured, and patients were classified into either an increase group, which had an increase in deviation $\geq 5$ prism diopters after short-term PAT, or a no-change group. Analysis was performed to investigate the clinical factors influencing the increase in exodeviation after short-term PAT.

Results: Fifty patients (44.2\%) showed an increase $\geq 5$ prism diopters during distance or near fixation after short-term PAT compared to the previous PACT: 12 patients (10.6\%) showed an increment at distance fixation, and 45 patients (39.8\%) showed an increase at near fixation. At distance fixation, the increase-group had a significantly smaller maximum angle measured by PACT. At near distance, age at PAT, maximum distance angle, minimum distance angle, maximum near angle, minimum near angle, angle fluctuation at near, and IXT type showed significant associations with positive short-term PAT response. In the multivariate analysis, older age and smaller maximum near angle were significantly associated with positive short-term PAT response at near fixation.

Conclusions: Short-term PAT could be helpful in older IXT patients with a small maximum angle of deviation at near fixation to mitigate the vergence aftereffect and show the maximum angle of deviation.

Key Words: Intermittent exotropia, Monocular occlusion test, Prism adaptation test

Intermittent exotropia (IXT) is the most common form of childhood strabismus in Asian countries [1], character-

Received: March 27, 2020 Final revision: April 26, 2020

Accepted: May 26, 2020

Corresponding Author: Jae Ho Jung, MD, PhD. Department of Ophthalmology, Seoul National University College of Medicine, 101 Daehak-ro, Jongno-gu, Seoul 03080, Korea. Tel: 82-2-2072-1765, Fax: 82-2-741-3187,

E-mail: jaeho.jung@snu.ac.kr ized by gradual and progressive exodeviation that presents primarily during distance fixation or when the patient is inattentive $[2,3]$. IXT has been classified based on distance and near exodeviations, and assessment of true exodeviation is a critical part of classifying IXT and deciding on a surgical plan. However, evaluation of true deviation in IXT patients is difficult because some of the patients exhibit a vergence aftereffect. 
A vergence aftereffect in IXT has been reported by Cooper and Medow [4]. They described that the amount of exodeviation changes by disrupting favorable binocular single vision status. This change could be observed by applying monocular occlusion or using prisms to neutralize the angle of deviation; both methods eliminate the disparity vergence signal, thereby inducing an increase in deviation in patients who have a strong vergence aftereffect. This phenomenon has many synonyms, such as phoria adaption, prism adaptation, and tenacious proximal convergence [5-7]. Different methods have been employed to minimize the vergence aftereffect, such as monocular occlusion, outdoor measurements, measurement under increased luminance, and the prism adaptation test (PAT) [810]. Among these, monocular occlusion has been the most intensively studied and is considered the standard method. However, PAT has not been widely used and studied in IXT patients.

The purpose of the present study was to evaluate the prevalence of the prism adaptation response in patients with IXT using the short-term PAT and to assess the factors associated with the prism adaptation response in patients with IXT.

\section{Materials and Methods}

\section{Ethics statement}

A case-controlled retrospective analysis was performed on patients with IXT at Seoul National University Children's Hospital. This study was reviewed and approved by the institutional review board of Seoul National University Hospital (1904-047-1025), Seoul, South Korea. All study conduct adhered to the tenets of the Declaration of Helsinki. Patient consent was waived after institutional review board review as this study was conducted retrospectively with no identifying features of individual patients.

\section{Subjects}

Patients with IXT without prior surgical treatments were included. All subjects underwent a complete ophthalmologic examination. For each subject, cycloplegic refraction and best-corrected visual acuity were measured. Both near $(40 \mathrm{~cm})$ and distance $(3 \mathrm{~m})$ stereoacuity were measured us- ing the Preschool Randot test (Stereo Optical, Chicago, IL, USA) while the subject was wearing his or her best refractive correction. A "pass" at each level was defined as two of three correct responses. The prism alternate cover test (PACT) was performed at each visit with fixation targets at $1 / 3 \mathrm{~m}$ and $6 \mathrm{~m}$ by a single ophthalmologist (JHJ). We also evaluated an office-based control scale at the distance target, with a scale ranging from 0 (good control, exophoria) to 5 (poor control, manifest exotropia) [11]. The type of exotropia before PAT was classified based on the difference between angles of distance and near deviation. Basic-type exotropia was defined as a distance-near difference less than 10 prism diopters (PD). Divergence excess (DE)-type exotropia was defined as a distance-near difference $\geq 10$ PD, and both true- and pseudo-DE exotropia were included. Convergence insufficiency-type exotropia was defined as a near-distance difference $\geq 10$ PD. Angle fluctuation was defined as the difference between the maximum and minimum angles. The following information was also collected: age at PAT, sex, history of occlusion, and presence or absence of accompanying symptoms such as glare, grimace, and diplopia.

Subjects were excluded if they had any conditions that could affect contrast sensitivity, including amblyopia, anisometropia, refractive errors greater than \pm 3.00 diopters (D) or astigmatism of more than $\pm 1.50 \mathrm{D}$, constant exotropia, vertical deviation $>5 \mathrm{PD}$, paralytic or restrictive exotropia, and/or known global developmental or neurological abnormalities. Those who showed poor cooperation to the prism and alternate cover test were also excluded.

\section{Short-term PAT}

Short-term PAT was performed on all patients at variable time points during the follow-up period. Personally designed prism glasses were used to offset the exodeviation previously measured by PACT. Each set of prism glasses consisted of a base in Fresnel prisms (Fresnel Prism \& Lens, Eden Prairie, MN, USA) with a similar amount of PD for each eye. For example, to offset an exodeviation of $20 \mathrm{PD}$, a pair of prism glasses containing a 10 PD base in prisms in each eye was used. Prism glasses were manufactured for patients with exodeviations of $20 \mathrm{PD}(10+10), 25$ PD $(12+12), 30$ PD $(15+15), 35$ PD $(15+20)$ and $40 \mathrm{PD}$ $(20+20)$. The patients were encouraged to wear the prism glasses for more than 30 minutes. Post-prism adaptation 
measurements were obtained by measuring the exodeviations using the prism with PACT while wearing the prism glasses and then adding the total amount of PD of the glasses to the measured angle. Deviation after short-term PAT was compared with the maximum angle of deviation, which was the largest among the deviation angles measured across multiple PACT examinations. IXT patients were classified into two groups according to response to the short-term PAT at distance and near fixation: increase and no-change. The increase group was defined as patients that had an increase in deviation $\geq 5$ PD after short-term PAT compared with the maximum angle. Otherwise, the patients were assigned to the no-change group.

\section{Statistical analyses}

Data normality was tested by the Kolmogorov-Smirnov test. Based on data normality, continuous variables were compared by using Student's $t$-test or Mann-Whitney test. Categorical variables were analyzed by chi-square or Fisher's exact test. Logistic regression analysis was performed to investigate the clinical factors influencing the increase in exodeviation after short-term PAT, first using a univariate model and then using a multivariate model that included variables from the univariate model with $p<0.15$. All statistical analyses were performed with IBM SPSS Statistics ver. 22.0 (IBM Corp., Armonk, NY, USA), and probability values less than 0.05 were considered significant.

Table 1. Comparison of characteristics of patients with intermittent exotropia classified according to response to short-term prism adaptation test at distant fixation

\begin{tabular}{|c|c|c|c|}
\hline & Increase $(n=12)$ & No change $(\mathrm{n}=101)$ & $p$-value \\
\hline Age at PAT (yr) & $6.93 \pm 2.20$ & $6.38 \pm 2.05$ & $0.39^{*}$ \\
\hline Sex (male : female) & $7: 5$ & $50: 51$ & $0.56^{\dagger}$ \\
\hline History of occlusion (no : yes) & $9: 3$ & $48: 53$ & $0.07^{\dagger}$ \\
\hline Glare (absent : present) & $5: 7$ & $66: 34$ & $0.12^{\ddagger}$ \\
\hline Grimace (absent : present) & $10: 2$ & $87: 13$ & $1.00^{\ddagger}$ \\
\hline Diplopia (absent : present) & $12: 0$ & $97: 3$ & $1.00^{\ddagger}$ \\
\hline SE OD (D) & $-0.55 \pm 2.07$ & $-0.64 \pm 1.66$ & $0.80^{\S}$ \\
\hline SE OS (D) & $-0.73 \pm 2.33$ & $-0.64 \pm 1.54$ & $0.82^{\S}$ \\
\hline VA OD & $0.92 \pm 0.29$ & $0.93 \pm 0.26$ & $0.87^{\S}$ \\
\hline VA OS & $0.83 \pm 0.39$ & $0.92 \pm 0.27$ & $0.32^{\S}$ \\
\hline VA OU & $1.00 \pm 0.00$ & $0.96 \pm 0.20$ & $0.50^{\S}$ \\
\hline DF stereo (poor : good) & $5: 5$ & $56: 30$ & $0.49^{\ddagger}$ \\
\hline NF stereo (poor : good) & $0: 10$ & $6: 80$ & $1.00^{\ddagger}$ \\
\hline DF $\max (\mathrm{D})$ & $25.42 \pm 4.98$ & $29.31 \pm 4.86$ & $0.010^{*, \Pi}$ \\
\hline $\mathrm{DF} \min (\mathrm{D})$ & $25.42 \pm 4.98$ & $28.02 \pm 5.21$ & $0.10^{*}$ \\
\hline DF fluctuation (D) & $0.00 \pm 0.00$ & $1.29 \pm 2.46$ & $0.048^{\S, \Pi}$ \\
\hline NF $\max (\mathrm{D})$ & $27.50 \pm 6.96$ & $28.36 \pm 7.68$ & $0.71^{*}$ \\
\hline NF min (D) & $27.33 \pm 7.13$ & $26.51 \pm 8.05$ & $0.74^{*}$ \\
\hline NF fluctuation (D) & $0.17 \pm 0.58$ & $1.81 \pm 3.52$ & $0.10^{\S}$ \\
\hline CS difference & $1.36 \pm 1.29$ & $0.83 \pm 0.92$ & $0.16^{\S}$ \\
\hline Type of exotropia (B : DE : CI) & $11: 0: 1$ & $90: 9: 1$ & $0.17^{\ddagger}$ \\
\hline
\end{tabular}

$\mathrm{PAT}=$ prism adaptation test $\mathrm{SE}=$ spherical equivalent; $\mathrm{OD}=$ right eye; $\mathrm{D}=$ diopter; $\mathrm{OS}=$ left eye; VA = visual acuity; OU = both eyes; $\mathrm{DF}=$ angle at distance fixation; $\mathrm{NF}=$ angle at near fixation; $\mathrm{CS}=$ control scale; $\mathrm{B}=$ basic type exotropia (distance-near difference $<10 \mathrm{D}$ ); $\mathrm{DE}=$ divergence excess type exotropia (distance-near difference $\geq 10 \mathrm{D}$ ); CI = convergence insufficiency type exotropia (near-distance difference $\geq 10 \mathrm{D})$.

"Student's $t$-test; ${ }^{\dagger}$ Chi-square test; ${ }^{\dagger}$ Fisher's exact test; ${ }^{\S}$ Mann-Whitney test; ${ }^{\Pi} p<0.05$. 


\section{Results}

A total of 113 patients was enrolled in the study. The mean age of the subjects was $6.4 \pm 2.1$ years (range, 2.112.3). The average maximum angle of deviation was $28.9 \pm$ 5.0 D at distance and $28.3 \pm 7.6 \mathrm{D}$ at near. The proportions of exotropia type were as follows: basic type $(n=101$, 89.4\%), DE-type ( $\mathrm{n}=9,8.0 \%)$, and convergence insufficiency-type $(\mathrm{n}=2,1.8 \%)$. One patient was unable to be classified due to the absence of angle measurement at near.

\section{Response to short-term PAT}

Fifty patients (44.2\%) showed an increase $\geq 5$ PD during distance or near fixation after short-term PAT compared to previous PACT: 12 patients (10.6\%) showed increment at distance fixation, and 45 patients (39.8\%) showed an increase at near fixation. Patients' characteristics and demographics are presented in Table 1 and 2 .

\section{Clinical characteristics based on short-term PAT}

In regard to short-term PAT at distance fixation, the increase-group had a significantly smaller maximum angle measured by PACT $(25.42 \pm 4.98 \mathrm{D})$ at distance than the no-change group $(29.31 \pm 4.86 \mathrm{D}, p=0.010)$. Other ophthalmic parameters showed no significant difference between the two groups (Table 1).

In terms of short-term PAT at near fixation, the increase-group ( $7.28 \pm 2.04$ years) was significantly older

Table 2. Comparison of characteristics of patients with intermittent exotropia classified according to response to short-term prism adaptation test at near fixation

\begin{tabular}{|c|c|c|c|}
\hline & Increase $(n=45)$ & No change $(n=68)$ & $p$-value \\
\hline Age at PAT (yr) & $7.28 \pm 2.04$ & $5.88 \pm 1.89$ & $<0.001^{*, \dagger}$ \\
\hline Sex (male : female) & $26: 19$ & $31: 37$ & $0.21^{\ddagger}$ \\
\hline History of occlusion (no : yes) & $25: 20$ & $32: 36$ & $0.44^{\ddagger}$ \\
\hline Glare (absent : present) & $26: 19$ & $45: 22$ & $0.31^{\ddagger}$ \\
\hline Grimace (absent : present) & $40: 5$ & $57: 10$ & $0.56^{\ddagger}$ \\
\hline Diplopia (absent : present) & $44: 1$ & $65: 2$ & $1.00^{\S}$ \\
\hline SE OD (D) & $-0.81 \pm 1.63$ & $-0.51 \pm 1.75$ & $0.40^{\dagger}$ \\
\hline SE OS (D) & $-0.70 \pm 1.65$ & $-0.61 \pm 1.64$ & $0.80^{\dagger}$ \\
\hline VA OD & $0.98 \pm 0.15$ & $0.90 \pm 0.31$ & $0.06^{\dagger}$ \\
\hline VA OS & $0.96 \pm 0.21$ & $0.88 \pm 0.33$ & $0.14^{\dagger}$ \\
\hline VA OU & $1.00 \pm 0.00$ & $0.94 \pm 0.24$ & $0.045^{*, \dagger}$ \\
\hline DF stereo (poor : good) & $22: 19$ & $39: 16$ & $0.08^{\ddagger}$ \\
\hline NF stereo (poor : good) & $3: 38$ & $3: 52$ & $1.00^{\S}$ \\
\hline DF $\max (\mathrm{D})$ & $27.09 \pm 4.74$ & $30.09 \pm 4.84$ & $0.002^{*, \dagger}$ \\
\hline $\mathrm{DF} \min (\mathrm{D})$ & $26.33 \pm 4.88$ & $28.68 \pm 5.28$ & $0.019^{*, \dagger}$ \\
\hline DF fluctuation (D) & $0.78 \pm 1.94$ & $1.40 \pm 2.58$ & $0.15^{\dagger}$ \\
\hline NF $\max (\mathrm{D})$ & $23.95 \pm 8.58$ & $31.06 \pm 5.28$ & $<0.001^{*, \dagger}$ \\
\hline $\mathrm{NF} \min (\mathrm{D})$ & $23.34 \pm 8.67$ & $28.71 \pm 6.67$ & $<0.001^{*, \uparrow}$ \\
\hline NF fluctuation (D) & $0.60 \pm 1.60$ & $2.32 \pm 4.02$ & $0.002^{*, \dagger}$ \\
\hline CS difference & $1.10 \pm 1.14$ & $0.75 \pm 0.82$ & $0.16^{\Pi}$ \\
\hline Type of exotropia (B : DE : CI) & $36: 8: 0$ & $65: 1: 2$ & $0.001^{*, \S}$ \\
\hline
\end{tabular}

$\mathrm{PAT}=$ prism adaptation test $\mathrm{SE}=$ spherical equivalent; $\mathrm{OD}=$ right eye; $\mathrm{D}=$ diopter; $\mathrm{OS}=$ left eye; $\mathrm{VA}=$ visual acuity; OU = both eyes; $\mathrm{DF}=$ angle at distance fixation; $\mathrm{NF}=$ angle at near fixation; $\mathrm{CS}=$ control scale; $\mathrm{B}=$ basic type exotropia (distance-near difference $<10 \mathrm{D}$ ); $\mathrm{DE}=$ divergence excess type exotropia (distance-near difference $\geq 10 \mathrm{D}$ ); CI = convergence insufficiency type exotropia (near-distance difference $\geq 10 \mathrm{D}$ ).

${ }^{*} p<0.05 ;{ }^{\dagger}$ Student's $t$-test; ${ }^{\star}$ Chi-square test; ${ }^{\circledR}$ Fisher's exact test; ${ }^{\Pi}$ Mann-Whitney test. 
than the no-change group $(5.88 \pm 1.89$ years, $p<0.001)$. The maximal and minimal angles measured by PACT, in both at distance and near, were significantly smaller in the increase-group compared to the no-change group (Table 2). The angle fluctuation was significantly lower in the increase-group $(0.60 \pm 1.60 \mathrm{D})$ than in the no-change group $(2.32 \pm 4.02 \mathrm{D}, p=0.002)$. The IXT type composition significantly differed between the two groups (Table 3 ).

\section{Short-term PAT response factors}

Because the short-term PAT response was more pro- nounced at near fixation, factors that influenced the shortterm PAT response at near fixation were investigated. In the univariate model, age at PAT ( $p<0.001)$, maximum distance angle ( $p=0.003)$, minimum distance angle ( $p=$ $0.023)$, maximum near angle $(p<0.001)$, minimum near angle ( $p=0.001)$, angle fluctuation at near $(p=0.014)$, and IXT type ( $p=0.047)$ showed significant associations with positive short-term PAT response (Table 3).

The multivariable analysis included variables from the univariate model with $p<0.15$ : age, maximum and minimum distance angles, maximum and minimum near angles, maximum control scale, inter-eye difference of con-

Table 3. Factors that affected a significant increase after short-term prism adaptation test at near fixation

\begin{tabular}{|c|c|c|c|c|c|c|}
\hline & \multicolumn{3}{|c|}{ Univariate analysis } & \multicolumn{3}{|c|}{ Multivariate analysis } \\
\hline & OR & $\begin{array}{l}\text { 95\% confidence } \\
\text { interval }\end{array}$ & $p$-value & OR & $\begin{array}{l}\text { 95\% confidence } \\
\text { interval }\end{array}$ & $p$-value \\
\hline Age at PAT & 1.433 & $1.162-1.768$ & $<0.001$ & 1.565 & $1.187-2.063$ & $0.002^{*}$ \\
\hline Sex (male : female) & 0.612 & $0.286-1.309$ & 0.21 & & & \\
\hline History of occlusion & 0.741 & $0.346-1.585$ & 0.44 & & & \\
\hline Glare & 1.495 & $0.685-3.264$ & 0.31 & & & \\
\hline Grimace & 0.713 & $0.226-2.244$ & 0.56 & & & \\
\hline Diplopia & 0.739 & $0.065-8.396$ & 0.81 & & & \\
\hline SE OD & 0.892 & $0.700-1.136$ & 0.35 & & & \\
\hline SE OS & 0.938 & $0.734-1.199$ & 0.61 & & & \\
\hline VA OU & 6.211 & $0.947-40.716$ & 0.06 & & & \\
\hline DF stereo (poor : good) & 2.105 & $0.904-4.905$ & 0.09 & & & \\
\hline NF stereo (poor : good) & 0.731 & $0.140-3.821$ & 0.71 & & & \\
\hline DF max & 0.877 & $0.805-0.956$ & 0.003 & 1.304 & $0.849-2.003$ & 0.23 \\
\hline DF min & 0.913 & $0.845-0.987$ & 0.023 & 0.951 & $0.630-1.435$ & 0.81 \\
\hline DF fluctuation & 0.879 & $0.731-1.057$ & 0.17 & & & \\
\hline NF max (D) & 0.843 & $0.778-0.914$ & $<0.001$ & 0.555 & $0.352-0.873$ & $0.011^{*}$ \\
\hline NF $\min (\mathrm{D})$ & 0.908 & $0.857-0.962$ & 0.001 & 1.354 & $0.907-2.022$ & 0.14 \\
\hline NF fluctuation & 0.787 & $0.650-0.954$ & 0.014 & & & \\
\hline CS difference & 1.444 & $0.954-2.186$ & 0.08 & 1.184 & $0.664-2.110$ & 0.57 \\
\hline CS maximum & 0.715 & $0.465-1.101$ & 0.13 & 0.794 & $0.436-1.446$ & 0.45 \\
\hline Type of exotropia & & & 0.047 & & & 0.80 \\
\hline $\mathrm{B}$ & Ref & & & Ref & & \\
\hline $\mathrm{DE}$ & 14.444 & $1.737-120.141$ & 0.013 & 0.283 & $0.007-11.139$ & 0.50 \\
\hline $\mathrm{CI}$ & 0.000 & 0.000 & 1.00 & 0.000 & 0.000 & 1.00 \\
\hline
\end{tabular}

$\mathrm{OR}=$ odds ratio; PAT = prism adaptation test; $\mathrm{SE}=$ spherical equivalent; $\mathrm{OD}=$ right eye; $\mathrm{OS}=$ left eye; $\mathrm{VA}=$ visual acuity; $\mathrm{OU}=$ both eyes; $\mathrm{DF}=$ angle at distance fixation; $\mathrm{NF}=$ angle at near fixation; $\mathrm{D}=$ diopter; $\mathrm{CS}=$ control scale; $\mathrm{B}=$ basic type exotropia $($ distance-near difference $<10 \mathrm{D}$ ); $\mathrm{DE}=$ divergence excess type exotropia (distance-near difference $\geq 10 \mathrm{D}$ ); $\mathrm{CI}=$ convergence insufficiency type exotropia (near-distance difference $\geq 10 \mathrm{D}$ ). ${ }^{*} p<0.05$. 
trol scale, and IXT type. In the multivariate analysis, older age $(\mathrm{OR}=1.565, p=0.002)$ and smaller maximum near angle $(\mathrm{OR}=0.555, p=0.011)$ were significantly associated with a positive short-term PAT response (Table 3).

\section{Discussion}

Previous studies have demonstrated that PATs can increase the angle of exodeviation at distance and near fixation [12-15]. However, the PAT did not elicit a response in all IXT patients. Our results showed that among 113 patients, a total of 50 patients (44.2\%) with exotropia showed an increase $\geq 5$ PD after short-term PAT. Twelve patients $(10.6 \%)$ showed an increase at distance fixation, while 45 patients $(39.8 \%)$ showed an increase at near fixation. This trend is consistent with a previous study showing that the percentage of prism adaptation responders at near fixation was greater than that of those that showed a prism adaptation response at distance fixation, at $27 \%$ and $10 \%$, respectively [15].

At distance fixation, a smaller angle of deviation measured by PACT and a lower angle variability among multiple PACT examinations were associated with a short-term PAT response. Patient age, sex, presence of symptoms associated with IXT, degree of binocularity, and type of exotropia were not associated with short-term PAT response. In contrast, in a report by Ohtsuki et al. [13], younger patients with larger initial angle of deviation showed a marked response to prism correction at distant fixation. However, Ohsuki et al. [13] only included basic type of IXT patients and compared the age of the patients at the time of the surgery, rather than the age at which PAT was applied. These discrepancies may have led to different results. At near fixation, in the univariate model, age at the PAT, maximum distance angle, minimum distance angle, maximum near angle, minimum near angle, and angle fluctuation at near showed significant associations with increased exodeviation after short-term PAT. The patients with DE-type exotropia demonstrated a tendency toward an increase in exodeviation after the PAT. In the multivariate model, older age and smaller near angle with PACT were significantly correlated with increase in exodeviation after short-term PAT. Our findings suggest that short-term PAT could be useful in older patients with a small maximum angle of deviation at near fixation.
Postoperative results for IXT can sometimes be discouraging for patients due to the high rate of recurrence over time [16]. Pritchard attributed this finding to variability in the measurement of exotropia and, consequently, not operating to the full angle of deviation [17]. Thus, the current trend is to target the largest angle for optimal surgical results $[9,18]$. To do so, the vergence aftereffect, a temporary change in eye position that results from any sustained vergence and does not dissipate with a brief PACT, should be evaluated before surgery $[4,9]$. Different methods have been employed to exclude these effects and determine the latent exodeviation, such as monocular occlusion, outdoor measurements, measurement under increased luminance, and PAT [8,13,18-22]. Among these, monocular occlusion has been the most widely used and studied. The monocular occlusion test can reveal latent exodeviation by blocking fusion stimuli and disrupting convergence tonus, which has been shown to significantly increase the angle of exodeviation at distance and near fixation $[10,19]$. However, monocular occlusion has practical disadvantages, which include challenges with maintaining dissociation during the post-occlusion examination, as fusion often occurs while removing the subject's occlusion patch. In addition, skin irritation due to adhesives used in the occluding stickers can be another problem. In comparison, in addition to causing no skin irritation, short-term PAT offers a more convenient and patient-friendly way to measure the latent angle, by simply adding prisms in front of prism glasses, without the hassle of maintaining dissociation during occlusion patch removal.

There is currently no consensus on the appropriate duration for PAT. In 1997, Ohtsuki et al. [13] performed PAT for three to five days, while in 2001, the duration was reduced to two to three hours at 15- to 30-minute intervals. Dadeya et al. [12] performed PAT for two weeks. Kiyak Yilmaz et al. [14] performed PAT for three or four hours until no additional prism was required to neutralize the deviation. Our results with the short-term PAT response at both near and distance fixation are similar to those of Ohtsuki et al. [15], which imply that a short term PAT of 30 minutes may be sufficient. In terms of esotropia, the conventional PAT is time consuming, which has led to decreased feasibility in clinical practice [23,24]. Alternatively, Akbari et al. [25] prospectively studied PAT with a short time frame in esotropic patients and found that, of the 14 subjects, those who showed motor stability after 20 minutes exhibited no fur- 
ther increase in deviation after 1 hour. However, the mechanism through which PAT revealed the latent angle may differ between exotropia and esotropia patients. To determine the optimal duration of PAT, further investigation for the variable duration of PAT is warranted.

The present study has several limitations. First was the retrospective design. A limited number of subjects and limited angle of deviations were included. Also, accommodative convergence was not measured. However, the underlying mechanism of vergence aftereffect differs from that of accommodative convergence; vergence aftereffect is a slow adaptation mechanism and is operated by different neurologic mechanisms compared to accommodative convergence [7]. Additionally, we were unable to determine whether short-term PAT could be an alternative option to monocular occlusion testing in patients with IXT. We expect further investigations to determine the results and parameters for this question.

In conclusion, short-term PAT may be useful in older patients with a small maximum angle of deviation at near fixation. Short-term PAT could be helpful in these patients to mitigate vergence aftereffect and to reveal the maximum angle of deviation regardless of the type of exotropia.

\section{Conflict of Interest}

No potential conflict of interest relevant to this article was reported.

\section{References}

1. Mohney BG, Huffaker RK. Common forms of childhood exotropia. Ophthalmology 2003;110:2093-6.

2. Jampolsky A. Differential diagnostic characteristics of intermittent exotropia and true exophoria. Am Orthopt $J$ 1954;4:4855.

3. von Noorden G. Binocular vision and ocular motility. 6th ed. St Louis: CV Mosby; 2002. p. 356-76.

4. Cooper J, Medow N. Major review: Intermittent exotropia basic and divergence excess type. Binocul Vis Eye Muscle Surg $Q$ 1993;8:185-216.

5. Kushner BJ. Selective surgery for intermittent exotropia based on distance/near differences. Arch Ophthalmol 1998;116:324-8.
6. Schor CM. The influence of rapid prism adaptation upon fixation disparity. Vision Res 1979;19:757-65.

7. Brodsky MC. Phoria adaptation: the ghost in the machine. J Binocul Vis Ocul Motil 2020;70:1-10.

8. Burian HM. Exodeviations: their classification, diagnosis and treatment. Am J Ophthalmol 1966;62:1161-6.

9. Kushner BJ. Exotropic deviations: a functional classification and approach to treatment. Am Orthopt $J$ 1988;38:8193.

10. Scobee RG. Exophoria: the oculorotary muscles. St Louis: CV Mosby; 1952. p. 171.

11. Mohney BG, Holmes JM. An office-based scale for assessing control in intermittent exotropia. Strabismus 2006;14:14750.

12. Dadeya S, Kamlesh, Naniwal S. Usefulness of the preoperative prism adaptation test in patients with intermittent exotropia. J Pediatr Ophthalmol Strabismus 2003;40:85-9.

13. Ohtsuki H, Hasebe S, Okano M, Furuse T. Comparison of surgical results of responders and non-responders to the prism adaptation test in intermittent exotropia. Acta Ophthalmol Scand 1997;75:528-31.

14. Kiyak Yilmaz A, Kose S, Guven Yilmaz S, Uretmen O. The impact of prism adaptation test on surgical outcomes in patients with primary exotropia. Clin Exp Optom 2015;98:2247.

15. Ohtsuki H, Hasebe S, Kono R, et al. Prism adaptation response is useful for predicting surgical outcome in selected types of intermittent exotropia. Am J Ophthalmol 2001;131:117-22.

16. Clarke WN, Noel LP. Surgical results in intermittent exotropia. Can J Ophthalmol 1981;16:66-9.

17. Pritchard C. Intermittent exotropia: how do they "turn out"? Am Orthopt J 1993;43:60-6.

18. Kushner BJ. The distance angle to target in surgery for intermittent exotropia. Arch Ophthalmol 1998;116:189-94.

19. Gurlu VP, Erda N. Diagnostic occlusion test in intermittent exotropia. J AAPOS 2008;12:504-6.

20. Han JM, Yang HK, Hwang JM. Efficacy of diagnostic monocular occlusion in revealing the maximum angle of exodeviation. Br J Ophthalmol 2014;98:1570-4.

21. Burian HM, Spivey BE. The surgical management of exodeviations. Am J Ophthalmol 1965;59:603-20.

22. Wirtschafter J, von Noorden G. The effect of luminance on exodeviation. Invest Ophthalmol 1964;3:549.

23. Repka MX, Connett JE, Scott WE. The one-year surgical outcome after prism adaptation for the management of acquired esotropia. Ophthalmology 1996;103:922-8. 
24. Kutschke PJ, Keech RV. Surgical outcome after prism adaptation for esotropia with a distance-near disparity. $J A A$ POS 2001;5:189-92.

25. Akbari MR, Mehrabi Bahar MR, Mirmohammadsadeghi A, et al. Short prism adaptation test in patients with acquired nonaccommodative esotropia: clinical findings and surgical outcome. J AAPOS 2018;22:352-5. 